\title{
An Artificial Intelligence Prediction Method of Bottomhole Flowing Pressure for Gas Wells Based on Support Vector Machine
}

\author{
Qin-Feng DI ${ }^{1, a}$, Wei $\mathrm{CHEN}^{1}$, Jing-Nan $\mathrm{ZHANG}^{1}$, Wen-Chang WANG ${ }^{2, a}$ and \\ Hui-Juan CHEN² \\ ${ }^{1}$ Shanghai Institute of Applied Mathematics and Mechanics, Shanghai University, Shanghai 200072 , \\ China \\ 2 Shanghai Key Laboratory of Mechanics in Energy Engineering, Shanghai 200072, China
}

Keywords: Flowing bottomhole pressure, Support vector machine, Random samples selection, Gas wells.

\begin{abstract}
The flowing bottomhole pressure (FBHP) of gas wells was affected by many factors. Although a lot of research works have been done to predict the FBHP and at least more than ten models were proposed, but no one can effectively provide an accurate results for all ranges of production data and conditions due to the existence of many uncertain relations between the changeable influence factors. In this paper, an artificial intelligence prediction method for FBHP based on the support vector machine (SVM), named the FBHP-SVM method, was studied, and a support vector regression (SVR) model with $\varepsilon$-insensitive loss function ( $\varepsilon$-SVR) based on radial basis function (RBF) was used to predict the FBHP of gas wells. Compared with the true values, the average absolute and relative errors of the new method were $0.27 \mathrm{MPa}$ and $2.29 \%$, respectively. The FBHP-SVM method was also compared to the vertical pipe flowing method. The results showed this new method was a new practical tool to predict FBHP in gas wells and it had a satisfying prediction accuracy.
\end{abstract}

\section{Introduction}

Accurately predicting the flowing bottomhole pressure (FBHP) of gas wells is the basis of dynamic analysis and production strategies optimization. The FBHP prediction is very complicated because many parameters have influence on the FBHP in the wellbore and they are continuous changing in the process of production. Although the first empirical formula has been put forward for many decades and many new methods have been proposed continually, there is no an applicable available model due to the existence of many uncertain relations between the changeable influence factors [1]. These prediction methods can be roughly classified into three kinds, i.e. empirical correlations, mechanistic models and artificial intelligence methods.

Many empirical correlations for FBHP prediction have been developed since the early 1940s. Most of these correlations were proposed by the investigators from laboratory studies, including those of Duns and Ros [2], Hagedorn and Brown [3], Beggs and Brill [4] and Orkiszewski [5]. Generally, these empirical correlations had a perfect performance under the condition that the model was proposed. But when the prediction conditions differ from the specific boundaries that the model was proposed, the FBHP prediction accuracy tends to decrease. Under this circumstances, the mechanistic models were developed to predict FBHP for gas wells. Most of them were semiempirical models and had sound theoretical foundation and wider application than empirical correlations [6]. The widely used mechanistic models are those of Rzasa and Katz [7], Cullender and Smiths [8], Ansari et al. [9], Chokshi et al. [10], Hasan and Kabir [11]. However, some empiricism, more or less, are still involved to overcome the complexity of the problem and the mechanistic models are very difficult to meet the requirement of a complicated well in which the continuous changes of the temperature and pressure will directly affect the gas production rate. And the gradual change in gas volumes will lead to the change of liquid slip velocity and the appearance of new flow patterns. The variation of flow patterns and their transition boundaries inside the well bore 
contributed to the deviation in FBHP predictions. In this condition, in order to decrease the deviations, the most suitable mechanistic model for each of these flow patterns needs to be used to predict the FBHP, which adds the complexity of calculation process. Thus, a method that can be applied to all ranges of production and conditions is urgent to be studied, and without doubt, new approaches that go beyond the conventional analysis methods are good choices.

In the recent few decades, the artificial intelligence methods are frequently used in oil and gas industries, and their unique advantages of solving various difficult engineering problems have drawn a great deal of attention. To overcome above-mentioned shortcomings associated with the empirical correlations and mechanistic models, researchers made use of artificial intelligence-based methods foremost of which was the classical artificial neural network (ANN) and its variants. But still, ANN had several inherent drawbacks such as over fitting, slow convergence, poor generalizing performance, and arriving at local minimum, and so on [12]. In order to achieve this target, a new type of artificial intelligence tool based on support vector machine (SVM) was utilized to make FBHP prediction in this paper. SVM is a new type of machine learning algorithm. It predicts unknown data by self-learning of the known data and can obtain the inherent laws even if it cannot be gotten by theory analysis. In addition, the SVM method has many unique advantages, such as strong generalization and convergence to global optimization, and dimensional insensitivity [13]. All of these characteristics make it possible to achieve the FBHP prediction no matter how complicated the conditions are, even in directional wells or horizontal wells. The works in this paper focus on vertical wells, and include four aspects. Firstly, a model based on radial basis function was used to establish the FBHP prediction model of gas wells and a FBHP prediction program was developed with MATLAB. Secondly, the effectiveness of the model and the program were tested by an exponential function with two independent variables and a noise item. Thirdly, the field data collected from Sulige gas field were used to evaluate the prediction accuracy based on random selection of the verification sample. Finally, the reliability of new developed method was verified by comparing with an existing widely used mechanistic model.

\section{Methodology of Support Vector Method}

SVM was proposed by Vapnik in 1995 based on the structural risk minimization principle and was an effective machine learning method for classification problems, especially for small sample of training vectors [14]. It studies how to construct learning machine, to realize the pattern classification. It aims to seek an optimal hyperplane that correctly classifies data points as much as possible and separates the points of two classes as far as possible [15]. SVM is considered as a highly efficient better performance classifier, so that it can replace the multi-layer perceptron, radial basis function (RBF) neural network and the polynomial network and so on other existing learning algorithm [16].

\section{Support Vector Regression}

The support vector regression (SVR) is the application of the SVM on regression problems and is based on the theory of linear regression in a multidimensional feature space [17]. The optimal hyperplane of SVR is to make the overall deviation between the predicted values and the true values minimum [18].

In the linearly separable case, Fig.1 shows the example of one-dimensional linear regression function with $\varepsilon$ insensitive region. The variable $\xi$ measures the error of the training point and the error is zero in $\varepsilon$ insensitive region. In the nonlinearly separable case, the input data $x$ can be transformed into the higher dimensional feature space where $x$ is linearly separable by using a kernel function. The support vector regression model with $\varepsilon$ insensitive region is named $\varepsilon-S V R$ [19]. 


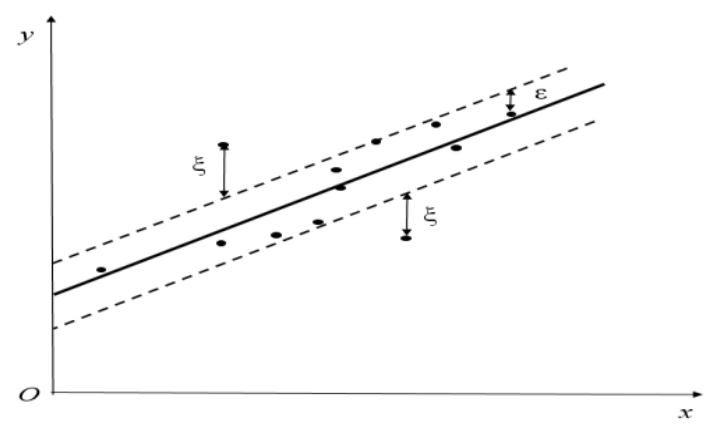

Figure 1. One-dimensional linear regression function with $\varepsilon$ insensitive region [19].

Assuming a dataset $T=\left\{\left(x_{i}, y_{i}\right), i=1, \ldots, n\right\}$, wherein the independent variables are $x_{i} \in R^{d}(d$ dimensionality) and the related dependent variables are $y_{i} \in R$, an objective function $f(x)$ needs to be found to predict the corresponding function values of any independent variables. Because the $\varepsilon$-SVR model can be converted into a dual problem of a convex quadratic program optimization, the dual problem can be represented as the maximizing function of Eq. (1) under the constraints of Eq. (2) [20].

$$
W\left(\alpha, \alpha^{*}\right)=-\frac{1}{2} \sum_{i, j=1}^{n}\left(\alpha_{i}-\alpha_{i}^{*}\right)\left(\alpha_{j}-\alpha_{j}^{*}\right) K\left(x_{i}, x_{j}\right)+\sum_{i=1}^{n}\left(\alpha_{i}-\alpha_{i}^{*}\right) y_{i}-\sum_{i=1}^{n}\left(\alpha_{i}+\alpha_{i}^{*}\right) \varepsilon
$$

where $\alpha$ and $\alpha^{*}$ stand for the Lagrange multipliers; $n$ is the sample size; $i$ and $j$ represent the sample numbers and $K\left(x_{i}, x_{j}\right)$ stands for the kernel function [20].

$$
\begin{gathered}
\sum_{i=1}^{n}\left(\alpha_{i}-\alpha_{i}^{*}\right)=0 \\
0 \leq \alpha_{i} \leq C \quad, \quad 0 \leq \alpha_{i}^{*} \leq C, \quad i=1,2, \ldots, n
\end{gathered}
$$

where $C$ stands for the penalty factor. The objective function can be expressed as [20]:

$$
f(x)=\sum_{i=1}^{n}\left(\alpha_{i}-\alpha_{i}^{*}\right) K\left(x_{i}, x_{j}\right)+b
$$

where $b$ is the classification threshold and can be obtained by the following equations [20]:

$$
\left\{\begin{array}{ll}
\varepsilon-y_{i}+f\left(x_{i}\right)=0, & \alpha \in(0, C) \\
\varepsilon+y_{i}-f\left(x_{i}\right)=0, & \alpha^{*} \in(0, C)
\end{array}, \quad i=1,2, \ldots, n\right.
$$

\section{Solving $\varepsilon$-SVR Model by Self-developed Program}

The SVM models can be solved as a quadratic program problem [18]. A self-developed program based on the $\varepsilon$-SVR model was used to predict the FBHP of gas wells, which is named as the FBHPSVM method in this paper. The block diagram was shown in Fig.2. In the prediction model, three parameters of the kernel parameter $\sigma$, insensitive factor $\varepsilon$ and penalty factor $C$ have significant effects on prediction results. After the initial ranges of the three parameters were determined, the web search method was used for parameters optimization. The parameters that accords with the minimum training sample mean square error (MSE) were selected to establish the model.

$$
\min _{\sigma, C, \varepsilon} \sqrt{\frac{1}{n} \sum_{i=1}^{n}\left(f\left(x_{i}\right)-y_{i}\right)^{2}}
$$

where $f\left(x_{i}\right)$ and $y_{i}$ are the predicted values and the true values, respectively. 


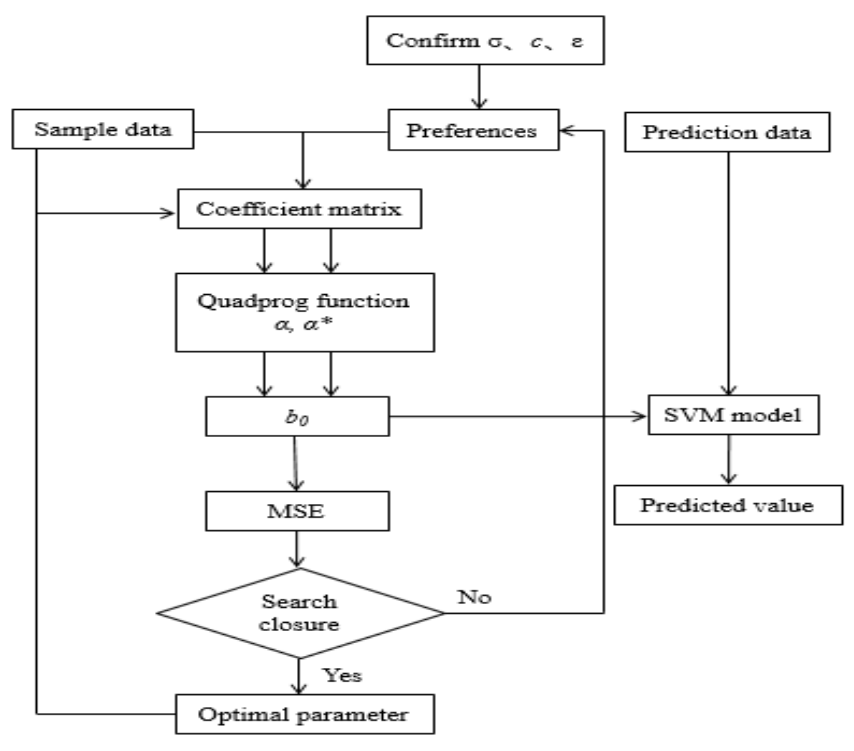

Figure 2. MATLAB program block diagram of the $\varepsilon$-SVR model.

\section{Method Verification}

A validity test must be performed in order to evaluate the new method. An exponential function with two independent variables and a noise item was used to test the effectiveness of the self-developed program. Assuming a test function $z=10 e^{-x^{2}-y^{2}}+\zeta$, wherein $\zeta$ is a normal distribution noise item and $\zeta \sim N(0,0.01)$. In $x \in[-1,1]$ and $y \in[-1,1], 121$ points were evenly selected to form the data set when the step-size is 0.2 , wherein 113 points were randomly picked as the training samples (TRS) and the remaining samples were as the verification samples (VES). The data set collected were showed in Fig.3. The initial ranges of three parameters were given as follows: $\sigma$ is between 0.1 and 10 and the step is $0.2 ; \varepsilon$ is between 0.1 and 3 and the step is $0.1 ; C$ is between 10 and 100 and the step is 10 . Inputting data into the self-developed program and optimizing the model parameters, a set of optimal parameters of $\sigma, \varepsilon$ and $C$ was determined, i.e. 1.1, 0.1 and 10, respectively, and the corresponding MSE of training samples is 0.2217. The comparison results between true values and prediction results of the FBHP-SVM method were shown in Fig.4 and the detailed data of verification samples were listed in Table 1.
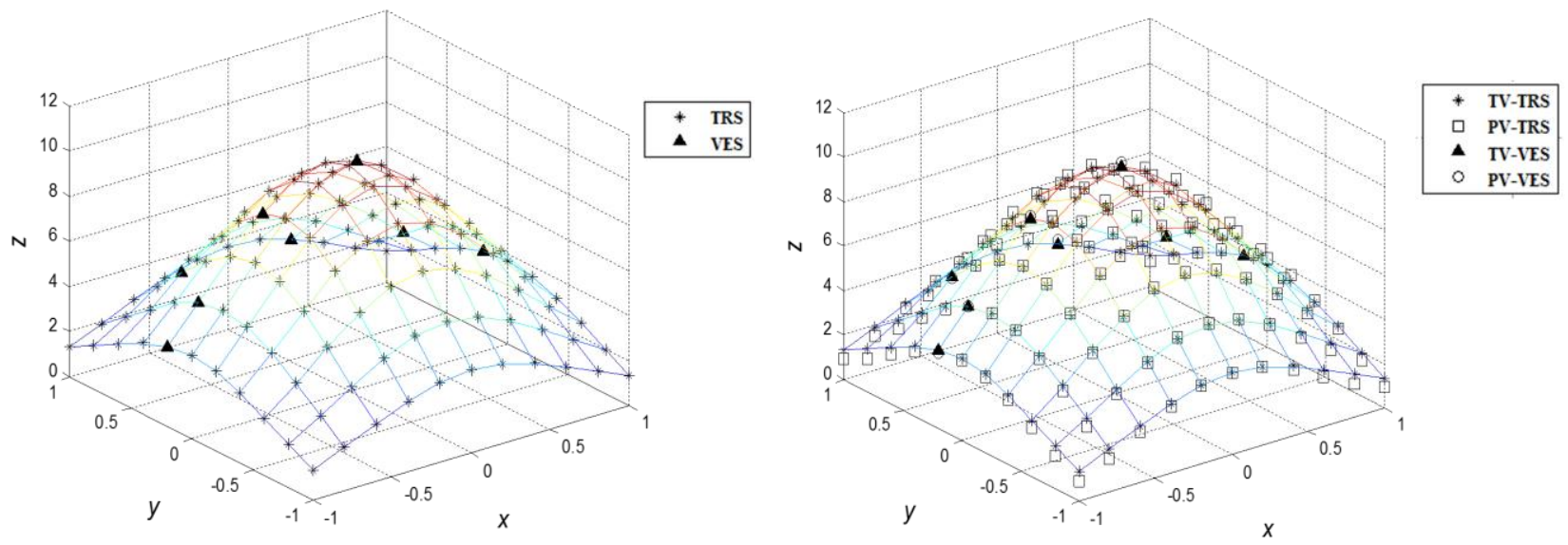

Figure 3. Division of samples collected.

Figure 4. Comparison between the true values and predicted values of the FBHP-SVM method. 
Table 1. Comparison between the true values and predicted values with verification samples.

\begin{tabular}{|c|c|c|c|c|c|}
\hline \multirow{2}{*}{$x$} & $y$ & \multicolumn{2}{|c|}{$z$} & & \\
\cline { 3 - 4 } & & True values & Predicted values & Absolute errors /MPa & Relative errors /\% \\
\hline-1 & 0.2 & 3.544 & 3.428 & 0.116 & 3.28 \\
\hline-0.8 & 0.2 & 5.072 & 5.060 & 0.012 & 0.24 \\
\hline-0.6 & 0.6 & 4.869 & 4.806 & 0.063 & 1.29 \\
\hline-0.4 & 0.2 & 8.192 & 8.291 & 0.099 & 1.21 \\
\hline 0.2 & 0.2 & 9.240 & 9.432 & 0.192 & 2.08 \\
\hline 0.4 & 1 & 3.137 & 3.312 & 0.175 & 5.58 \\
\hline 0.8 & 0.6 & 3.688 & 3.766 & 0.078 & 2.12 \\
\hline 1 & 0.2 & 3.544 & 3.820 & 0.275 & 7.76 \\
\hline
\end{tabular}

Figure 4 shows that the differences between the predicted values with training samples (PV-TRS) and the true values of training samples (TV-TRS) are small. From Table 1, it is clearly observed that the largest absolute and relative errors between the predicted values with verification samples (PVVES) and the true values of verification samples (TV-VES) are $0.275 \mathrm{MPa}$ and $7.76 \%$, respectively. It illustrates that the self-developed program can be applied to predict FBHP of gas wells.

\section{Data Collection and Pre-processing}

\section{Data Collection}

Sixteen groups of samples obtained from well testing were collected from Sulige gas field of China [21]. For an model, properly selecting the inputs is very important. Unnecessary and uncertain inputs may increase prediction errors. After studying the different empirical correlations and mechanistic models, the following parameters are selected as input data to predict FBHP, i.e. wellhead pressure, $P_{c}$, well depth, $H$, relative density of gas, $\gamma_{g}$, average temperature in the wellbore, $T$, gas compression factor, $\bar{Z}$ and gas rate, $q_{s c}$.

\section{Data Normalization}

In order to avoid the magnitude difference between the input parameters and eliminate the dimension influence of different parameters, some data pre-processing must be performed in order to train the model rapidly and with more accuracy. The input data are needed to be transformed into a unified form with normalization. The normalization formula is as follows:

$$
x_{i j}=\frac{2\left(x_{i j}-x_{j \min }\right)}{x_{j \max }-x_{j \min }}-1
$$

where $x_{i j}$ and $x_{i j}$ are the $j$ th influence factors of the $i$ th training sample before and after normalization; $x_{j \max }$ and $x_{j \min }$ are the maximum and minimum values of the $j$ th influence factor in all the training samples.

\section{FBHP Prediction of Gas Wells Based on SVM}

After the influence factors and the value ranges of $\sigma, \varepsilon$ and $C$ were determined, the $\varepsilon$-SVR model for FBHP prediction of gas wells was established. Five groups of samples, in turn, were randomly picked as the verification samples. For every group of verification sample, a model was firstly established by importing the remaining fifteen groups of samples into the self-developed program as training samples according to the principal of minimum training sample mean square error, then the prediction value of FBHP was obtained by inputting the wellhead pressure, well depth, relative density of gas, average temperature in the wellbore, gas compression factor and gas rate of the verification sample into this model. The results were shown in Table 2 and Fig. 5. 
Table 2 and Fig. 5 have shown a good concordance of the predicted values of five groups of verification samples obtained by the FBHP-SVM method (PV-SVM) with the true values of verification samples (TV-VES).The average absolute and relative errors of the five groups of verification samples are $0.27 \mathrm{MPa}$ and $2.29 \%$, respectively, which indicates that the FBHP-SVM method proposed in this paper can be used to predict the FBHP of gas wells with a satisfying accuracy.

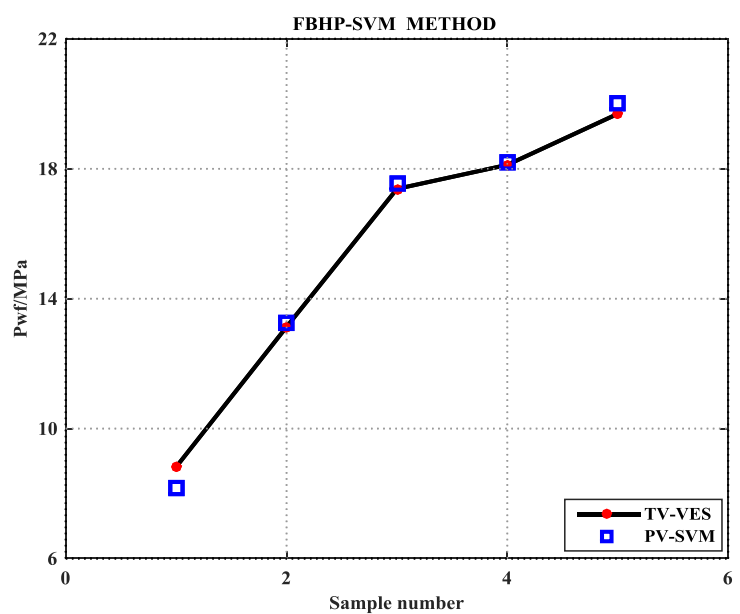

Figure 5. Comparison between the true values and predicted values of the FBHP-SVM method.

Table 2. Comparison between the true values and predicted values of the FBHP-SVM method

\begin{tabular}{|c|c|c|c|c|c|c|c|c|c|c|}
\hline \multirow{2}{*}{ 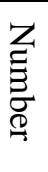 } & \multirow[b]{2}{*}{$\begin{array}{c}\mathrm{Ln}\left(P_{c}\right) \\
/ \mathrm{MPa}\end{array}$} & \multirow[b]{2}{*}{$H / \mathrm{m}$} & \multirow[b]{2}{*}{$\gamma_{g}$} & \multirow[b]{2}{*}{$\begin{array}{c}T \\
/{ }^{\circ} \mathrm{C}\end{array}$} & \multirow[b]{2}{*}{$\begin{array}{c}q_{s c} / \\
10^{4} \mathrm{~m}^{3} / \mathrm{d}\end{array}$} & \multirow[b]{2}{*}{$\bar{Z}$} & \multicolumn{2}{|c|}{$P_{w f} / \mathrm{MPa}$} & \multirow[b]{2}{*}{$\begin{array}{c}\text { Absolute } \\
\text { errors } \\
\text { /MPa }\end{array}$} & \multirow[b]{2}{*}{$\begin{array}{c}\text { Relative } \\
\text { errors } \\
1 \%\end{array}$} \\
\hline & & & & & & & $\begin{array}{c}\text { True } \\
\text { values }\end{array}$ & $\begin{array}{c}\text { Predicted } \\
\text { values }\end{array}$ & & \\
\hline 1 & 1.96 & 3273.4 & 0.6029 & 67.15 & 1.531 & 0.9873 & 8.82 & 8.15 & 0.67 & 7.60 \\
\hline 2 & 2.35 & 3292.9 & 0.6065 & 63.90 & 1.126 & 0.9942 & 13.13 & 13.24 & 0.11 & 0.84 \\
\hline 3 & 2.65 & 3331.7 & 0.6066 & 64.00 & 2.498 & 0.9873 & 17.38 & 17.54 & 0.16 & 0.92 \\
\hline 4 & 2.67 & 3335.0 & 0.6187 & 55.75 & 2.502 & 0.9869 & 18.12 & 18.19 & 0.07 & 0.39 \\
\hline 5 & 2.71 & 3312.9 & 0.6146 & 70.95 & 3.121 & 0.9915 & 19.69 & 20.02 & 0.33 & 1.68 \\
\hline \multicolumn{9}{|c|}{ Average error } & 0.27 & 2.29 \\
\hline
\end{tabular}

\section{Compared with the Vertical Pipe Flowing Method}

To demonstrate the superiority of the method proposed in this paper, the predicted results were compared with those predicted by the vertical pipe flowing method [22]. As a simplified mechanistic model, the vertical pipe flowing method's advantages are that it requires fewer required parameters and has a simple calculation process. Currently, vertical pipe flowing method is widely applied in the field.

\section{Vertical Pipe Flowing Method}

According to the pressure gradient equation in vertical gas wells, the expression for calculating FBHP by the vertical pipe flowing method under the condition of ignoring the kinetic pressure gradient is as follows [23]:

$$
p_{w f}=\sqrt{p_{c}^{2} e^{2 s}+1.32 \times 10^{-18} f\left(T \bar{Z} q_{s c}\right)^{2}\left(e^{2 s}-1\right) / D^{5}}
$$

where $P_{w f}$ is the FBHP, MPa; $P_{c}$ is the casing pressure, MPa; $f$ is the friction resistance, nondimensional; $\bar{T}$ is the average temperature in the wellbore, $\mathrm{K} ; \bar{Z}$ is the gas compression factor, nondimensional; $q_{s c}$ is the gas rate, $\mathrm{m}^{3} / \mathrm{d}$; and $D$ is the tube diameter, $\mathrm{m} . s$ is the exponent [23]. 


$$
s=\frac{0.03418 \gamma_{g} H}{\bar{T} \bar{Z}}
$$

where $\gamma_{g}$ is the relative density of gas, non-dimensional; and $H$ is the well depth, $\mathrm{m}$.

Because the friction factor $f$ varies with pipe surface roughness which is related to pipe material, manufacturing technology, corrosion and scaling condition, so it is very difficult to obtain accurate friction factor values. According to Wanglin's results, the friction factor can be ignored [17]. Then Eq. (7) can be written as follows:

$$
p_{w f}=p_{c} e^{\frac{0.03415 \gamma_{s} H}{T \bar{Z}}}
$$

Five groups of verification samples were used to calculate FBHP by the vertical pipe flowing method. The results are compared with the true values in Table 3 and Fig. 6.

From Table 3 and Fig. 6, it is clear that the average absolute and relative errors between the true values and predicted results with vertical pipe flowing method (PV-VP) are $0.40 \mathrm{MPa}$ and 2.39\%, respectively, which are in the acceptable range and can meet the needs of practical production.

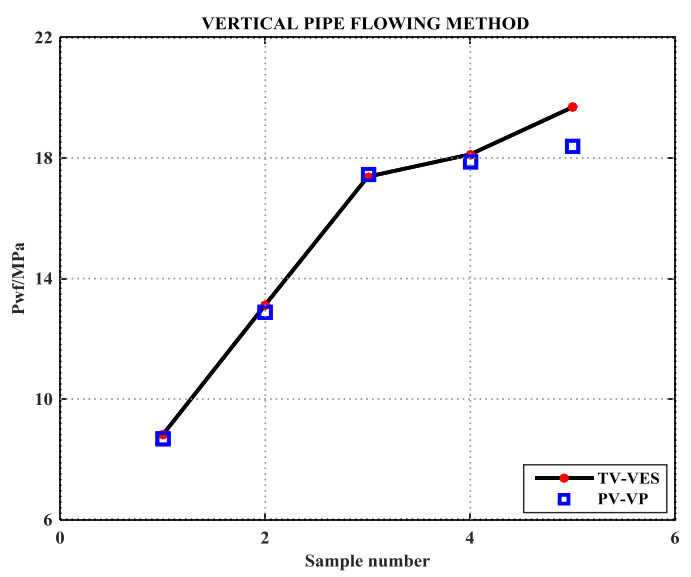

Figure 6. Comparison between true values and predicted values of vertical pipe flowing method.

\begin{tabular}{|c|c|c|c|c|c|c|c|c|c|}
\hline \multirow[b]{2}{*}{ Number } & \multirow{2}{*}{$\begin{array}{r}P_{C} / \\
\mathrm{MPa}\end{array}$} & \multirow[b]{2}{*}{$\begin{array}{l}H \\
/ \mathrm{m}\end{array}$} & \multirow[b]{2}{*}{$\gamma_{g}$} & \multirow{2}{*}{$\begin{array}{l}\bar{T} \\
/ \mathrm{K}\end{array}$} & \multirow[b]{2}{*}{$\bar{Z}$} & \multicolumn{2}{|c|}{$P_{w f} / \mathrm{MPa}$} & \multirow{2}{*}{$\begin{array}{c}\text { Absolute } \\
\text { errors } \\
/ \mathrm{MPa}\end{array}$} & \multirow{2}{*}{$\begin{array}{c}\text { Relative } \\
\text { errors } \\
1 \%\end{array}$} \\
\hline & & & & & & $\begin{array}{c}\text { True } \\
\text { values }\end{array}$ & $\begin{array}{c}\text { Predicted } \\
\text { values }\end{array}$ & & \\
\hline 1 & 7.10 & 3273.4 & 0.6029 & 340.15 & 0.9873 & 8.82 & 8.68 & 0.14 & 1.59 \\
\hline 2 & 10.50 & 3292.9 & 0.6065 & 336.9 & 0.9942 & 13.13 & 12.87 & 0.26 & 1.98 \\
\hline 3 & 14.20 & 3331.7 & 0.6066 & 337 & 0.9873 & 17.38 & 17.47 & 0.09 & 0.52 \\
\hline 4 & 14.40 & 3335 & 0.6187 & 328.75 & 0.9869 & 18.12 & 17.89 & 0.23 & 1.27 \\
\hline 5 & 15.00 & 3312.9 & 0.6146 & 343.95 & 0.9915 & 19.69 & 18.39 & 1.30 & 6.60 \\
\hline \multicolumn{8}{|c|}{ e error } & 0.40 & 2.39 \\
\hline
\end{tabular}

Table 3. Comparison between the true values and predicted values of vertical pipe flowing method

\section{Results Comparison and Analysis}

The comparison between the results obtained by the FBHP-SVM method and vertical pipe flowing method are shown in Table 4 and Fig. 8.

As Table 5 and Fig. 7 show, the average absolute errors between the true values and predicted results of the FBHP-SVM method and the vertical pipe flowing method, are $0.27 \mathrm{MPa}$ and $0.40 \mathrm{MPa}$ and the average relative errors are $2.29 \%$ and $2.39 \%$, respectively, both of which indicate that the FBHP-SVM method can be used as a new practical way to predict FBHP with satisfying accuracy. 
Especially, the prediction results of the vertical pipe flowing method are affected by the parameters in the equation (7) (9). When the pressure and temperature of formation change, the calculation formula or the value ranges of the friction resistance, $f$, the relative density of gas, $\gamma_{s}$ and the gas compression factor, $\bar{Z}$ will be different. Moreover, for directional wells or horizontal wells, the calculation method of the friction resistance, $f$ is also different from the vertical wells. Thus, for a complicated well, the prediction for FBHP calls for different methods for different regions of flow patterns and different deviation angles. The proposed FBHP-SVM method in this paper is applicable for all ranges of production conditions, so the FBHP-SVM method is a more practical tool to predict FBHP for gas wells.

Table 4. Comparison of results

\begin{tabular}{|c|c|c|c|c|c|c|c|}
\hline \multirow{3}{*}{ Samples } & \multicolumn{3}{|c|}{$P_{w f} / \mathrm{MPa}$} & \multicolumn{2}{c|}{ Absolute errors/MPa } & \multicolumn{2}{c|}{ Relative errors/\% } \\
\cline { 2 - 8 } & $\begin{array}{c}\text { True } \\
\text { values }\end{array}$ & $\begin{array}{c}\text { FBHP- } \\
\text { SVM } \\
\text { method }\end{array}$ & $\begin{array}{c}\text { Vertical pipe } \\
\text { flowing } \\
\text { method }\end{array}$ & $\begin{array}{c}\text { FBHP-SVM } \\
\text { method }\end{array}$ & $\begin{array}{c}\text { Vertical pipe } \\
\text { flowing } \\
\text { method }\end{array}$ & $\begin{array}{c}\text { FBHP- } \\
\text { SVM } \\
\text { method }\end{array}$ & $\begin{array}{c}\text { Vertical pipe } \\
\text { flowing } \\
\text { method }\end{array}$ \\
\hline 1 & 8.82 & 8.15 & 8.68 & 0.67 & 0.14 & 7.60 & 1.59 \\
\hline 2 & 13.13 & 13.24 & 12.87 & 0.11 & 0.26 & 0.84 & 1.98 \\
\hline 3 & 17.38 & 17.54 & 17.47 & 0.16 & 0.09 & 0.92 & 0.52 \\
\hline 4 & 18.12 & 18.19 & 17.89 & 0.07 & 0.23 & 0.39 & 1.27 \\
\hline 5 & 19.69 & 20.02 & 18.39 & 0.33 & 1.30 & 1.68 & 6.60 \\
\hline \multicolumn{3}{|c|}{ Average error } & 0.27 & 0.40 & 2.29 & 2.39 \\
\hline
\end{tabular}

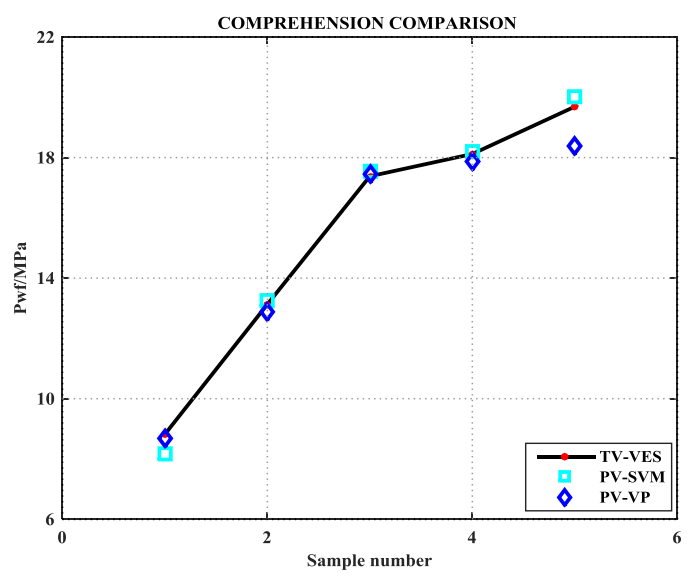

Figure 7. Results comparison.

\section{Conclusions}

The FBHP-SVM method was proposed in this paper to predict the FBHP of gas wells. The main conclusions are as follows.

(1) SVM method had been successfully used to predict the FBHP in this paper and a better performance had been gotten because it can suit all ranges of production conditions.

(2) In order to avoid human intervention, a random selection method of verification samples was used in the process of predicting FBHP of gas wells by the FBHP-SVM method. The results showed that the absolute and relative errors were $0.27 \mathrm{MPa}$ and $2.29 \%$, respectively, which indicated that this method can be used to predict flowing bottom hole pressure with a good prediction precision.

(3) The results showed that the FBHP-SVM method had an approximate accuracy compared with the vertical pipe flowing method. It is worth noting that this new method would have better application prospects because it does not rely on a given mechanistic model with changeable parameters and empirical factors. 


\section{Acknowledgement}

This research was supported in part by the National Science Funding of China (U1663205, 50874071), the Key Program of Science and Technology Commission of Shanghai Municipality (071605102), Shanghai Leading Academic Discipline Project (S30106), and the Shanghai Municipal Education Commission (Peak Discipline Construction Program).

\section{References}

1. Al-Shammari A. SPE Saudi Arabia Section Technical Symposium and Exhibition (Society of Petroleum Engineers, Al-Khobar, 2011)

2. Duns Jr H, Ros N C J. 6th World Petroleum Congress (Frankfurt am Main, 1963)

3. Hagedorn A R, Brown K E. Journal of Petroleum Technology. 17, 475 (1965)

4. Beggs D H, Brill J P. Journal of Petroleum technology. 25, 607 (1973)

5. Orkiszewski J. Journal of Petroleum Technology. 19, 829 (1967)

6. Osman E S A, Ayoub M A, Aggour M A. SPE Middle East Oil and Gas Show and Conference (Society of Petroleum Engineers, Kingdom of Bahrain, 2005)

7. Rzasa M J, Katz D L. Transactions of the AIME. 160, 100 (1945)

8. Cullender M H, Smith R V. Trans. AIME. 207, 281 (1965)

9. Ansari A M, Sylvester N D, Shoham O, et al. SPE Annual Technical Conference and Exhibition (Society of Petroleum Engineers, New Orleans, 1990)

10. Chokshi R N, Schmidt Z, Doty D R. SPE Western Regional Meeting (Society of Petroleum Engineers, Anchorage, 1996)

11. Hasan A R, Kabir C S. SPE. Production Engineering. 3, 263 (1988)

12. Xue X H, Yang X G, Chen X. Sci China Tech Sci. 57, 2379 (2014)

13. Zhang X G. Acta Automatica Sinica. 26, 32 (2000)

14. Liu H Q, Chen Q H, Li B, et al. Sci China Tech Sci. 54, 3119 (2011)

15. Farquad M A H, Ravi V, Raju S B. Expert Systems with Applications. 37, 5577 (2010)

16. Baydaroğlu Ö, Koçak K. Journal of Hydrology, 508, 356 (2014)

17. Li P F, Dong L M, Xiao H C, et al. Neurocomputing. 169, 34 (2015)

18. Di Q F, Hua S, Ding W P, et al. Journal of Hydrodynamics. Ser. B. 27, 99 (2015)

19. Bai P, Zhang X.B, Zhang B. Xi'an Electronic Sience and Technology University Press (Xi'an, 2008)

20. Chang C C, Lin C J. ACM Transactions on Intelligent Systems and Technology (TIST).2, 27 (2011)

21. Chen W. Xi'an Shiyou University (Master Thesis,2014)

22. Lin W, Peng C Z, Ni X W. Well Testing, 20, 25 (2011)

23. Yang, J S. Petroleum Industry Press (Beijing, 1992) 